\title{
A SECOND GRADIENT MODEL FOR DEFORMABLE POROUS MATRICES FILLED WITH AN INVISCID FLUID
}

\author{
Fransesco dell'Isola ${ }^{1}$ and Guilio Sciarra ${ }^{2}$ \\ ${ }^{1}$ Dip. Ingegneria Strutturale e Geotecnica, \\ ${ }^{2}$ Dip. Ingegneria Chimica, dei Materiali, delle Materie Prime e Metallurgia, \\ Università degli Studi di Roma "La Sapienza", Via Eudossiana 18, 00184 Roma, Italy
}

Romesh C. Batra

Dept. Engineering Sciences and Mechanics, Virginia Polytechnic Institute and State University, Blacksburg, VA 24060, USA

\begin{abstract}
In this paper we deal with an enlarged theory of binary mixtures: a second gradient solid constituent and a perfect fluid are considered. On the basis of this assumptions we obtain, for a linear elastic hollow cylinder, a set of density profiles of the solid matrix, parameterized by a suitable energetic coupling coefficient and characterized by the presence of boundary layers arising at the external surfaces of the body. A structural stability analysis of the partial differential equations, governing the motion of the mixture, is also developed, in a case which may be of interest in applications to underground structural engineering.
\end{abstract}

\section{Introduction}

A simplified model for mechanical systems constituted by a solid deformable porous matrix filled by a compressible fluid has been developed by [6], [2] and [10]. The main idea in their works stays in the "homogenization" assumption which leads us to accept the possibility of simultaneous placement of a solid and a fluid material particle at the same current place. In the literature when this assumption is accepted one talks about homogeneous mixtures. Observations of the behavior of fluid saturated solids have shown a not negligible increase of fluid percolation, through the pores of the solid matrix, with respect to the prediction provided by classical models of homogeneous mixtures (see e.g. [9]). In other words experimental evidence (see e.g. [3]) makes clear that the model of homogeneous mixture is not predictive in describing several phenomena occurring in fluid-saturated solids. A possible explanation for the 
previously described phenomena stays in the circumstances that the increase of percolation is not simply due to the increase of externally applied pressure but also to the pore-opening occurring in the vicinity of the boundary (see [3]). In this paper we do not directly deal with a micro-structured model, but consider a binary mixture model involving a second gradient solid and a perfect fluid; for the relationship between micro-structured and second gradient theories we refer to [7] and [8]. This kind of approach is very close to that based on the volume fraction concept, as this last reduces to the first one once a suitable constraint, among the enlarged set of state parameters, is assumed. On the basis of this approach and considering a linear elastic model, we study the equilibrium static configurations assumed by a porous hollow cylinder filled with a liquid. In particular assuming the energy functional to be split into a first gradient and a second gradient energy contribution, we address the parametric analysis of the equilibrium density profiles of the solid matrix with respect to a suitable energetic coupling coefficient between the solid and the fluid. Since we have chosen to deal with static deformations of the hollow cylinder we require the external tractions applied on both constituents to be conservative. Last but not least we discuss the structural stability properties of the governing equations, with respect to perturbations of the aforementioned coupling coefficient.

\section{Formulation of the Problem}

Material particles of the fluid and the solid are identified respectively by their position vectors $X_{f}$ and $X_{s}$ in fixed reference configurations $\Omega_{0}^{f}$ and $\Omega_{0}^{s}$. As is usually done in the theory of mixtures we presume that, at any time $t$, particles of both constituents may occupy the same position $\mathrm{x}$ in the present configuration $\Omega$. The velocity $\mathbf{v}_{\alpha}(\alpha=f, s)$ of the material particle $X_{\alpha}$ is defined by

$$
\mathbf{v}_{\alpha}=\frac{d_{\alpha} \mathbf{u}_{\alpha}\left(X_{\alpha}, t\right)}{d t}
$$

i.e. the material time derivative of the displacement of the $\alpha$-th constituent $\mathbf{u}_{\alpha}$ from its reference configuration.

Let $\rho_{f}$ and $\rho_{s}$ denote the apparent mass densities of the fluid and the solid then the mean velocity $\mathbf{v}$ of the mixture is defined by

$$
\rho \mathbf{v}=\rho_{f} \mathbf{v}_{f}+\rho_{s} \mathbf{v}_{s}
$$

Details of the theory of mixtures are given in [10], [9]. 


\section{Balance laws}

We use the principle of virtual power to derive the balance of linear momentum and the boundary conditions for each constituent. That is,

$$
\begin{aligned}
& \int_{\Omega}\left(\mathbf{m}_{s} \cdot \mathbf{v}_{s}+\mathbf{m}_{f} \cdot \mathbf{v}_{f}+\mathbf{T}_{s} \cdot \nabla \mathbf{v}_{s}-p_{f} \operatorname{div} \mathbf{v}_{f}+\mathbf{\Pi}_{s} \cdot \nabla \nabla \mathbf{v}_{s}\right) d V= \\
& =\int_{\Omega}\left(\mathbf{b}_{s} \cdot \mathbf{v}_{s}+\mathbf{b}_{f} \cdot \mathbf{v}_{f}\right) d V+\int_{\partial \Omega}\left(\mathbf{t}_{s} \cdot \mathbf{v}_{s}+\mathbf{t}_{f} \cdot \mathbf{v}_{f}+\boldsymbol{\tau}_{s} \cdot \frac{\partial \mathbf{v}_{s}}{\partial n}\right) d A
\end{aligned}
$$

Here $\mathbf{m}_{\alpha}$ is the bulk solid-fluid interaction force, $\mathbf{T}_{s}$ the partial Cauchy stress in the solid, $p_{f}$ the hydrostatic pressure in the perfect fluid, $\boldsymbol{\Pi}_{s}$ the secondorder stress in the solid, $\mathbf{b}_{\alpha}$ the density of partial body forces, $\mathbf{t}_{\alpha}$ the partial surface tractions, $\tau_{s}$ the traction corresponding to the second-order stress tensor in the solid and $\partial \mathbf{v}_{s} / \partial n$ the directional derivative of $\mathbf{v}_{s}$ along the outward unit normal $\mathbf{n}$ to the boundary $\partial \Omega$ of $\Omega$.

The physical meaning of $\boldsymbol{\Pi}_{s}$ and $\tau_{s}$ can be grasped in a way similar to that done in different contexts in [7] and [4]. We remark that the external action $\tau_{s}$ can be regarded as the sum of two different contributions, the first one is a doubly normal double force, i.e. an external areal action which works on the rate of opening, $\nabla \mathbf{v}_{s} \cdot \mathbf{n} \otimes \mathbf{n}$, of boundary pores along the outward unit normal $\mathbf{n}$, the other one a tangential couple working on the vorticity of the apparent velocity of the solid. This last areal action is considered in the Cosserat model for granular materials (see e.g. [5]) and in the present approach vanishes. The objectivity of the left hand side of (3) implies that the sum of the two internal supplies $\mathbf{m}_{f}$ and $\mathbf{m}_{s}$ of linear momentum equals zero and $\mathbf{T}_{s}$ is symmetric.

By using the divergence theorem and exploiting the fact that eqn.(3) must hold for all virtual velocities we obtain:

$$
\begin{aligned}
& \operatorname{div}\left(\mathbf{T}_{s}-\operatorname{div} \mathbf{\Pi}_{s}\right)-\mathbf{m}_{s}+\mathbf{b}_{s}=\mathbf{0}, \text { in } \Omega, \\
& -\nabla p_{f}-\mathbf{m}_{f}+\mathbf{b}_{f}=\mathbf{0}, \text { in } \Omega, \\
& \mathbf{m}_{s}+\mathbf{m}_{f}=\mathbf{0}, \text { in } \Omega, \\
& \quad\left(\mathbf{T}_{s}-\operatorname{div} \boldsymbol{\Pi}_{s}\right) \mathbf{n}-\operatorname{div}_{s}\left(\boldsymbol{\Pi}_{s} \mathbf{n}\right)=\mathbf{t}_{s}, \text { on } \partial \Omega, \\
& \quad\left(\boldsymbol{\Pi}_{s} \mathbf{n}\right) \mathbf{n}=\boldsymbol{\tau}_{s}, \text { on } \partial \Omega, \\
& -p_{f} \mathbf{n}=\mathbf{t}_{f}, \text { on } \partial \Omega,
\end{aligned}
$$

where $\operatorname{div}_{s}$ is the surface divergence on $\partial \Omega$.

\section{Constitutive relations}

The balance laws (4)-(5) are to be supplemented by constitutive relations; we express these in terms of the internal energy. We presume that the mixture is 
at a uniform temperature, the constituents are deforming quasi-statically so that their kinetic energy can be neglected, and no energy is dissipated. The internal energy density is split into two parts; a part that depends upon the "local" deformation of the solid and fluid particles and another part that depends upon a "nonlocal" measure of deformation of the solid particles: the latter is taken to be proportional to $\left|\nabla \rho_{s}\right|^{2}$. Thus we write the balance of internal energy as

$$
\begin{aligned}
& \frac{d_{\mathbf{v}}}{d t} \int_{\Omega} \rho\left[\epsilon\left(\rho_{f}, \mathbf{F}_{s}, X_{s}\right)+\frac{\lambda_{s}}{2 \rho}\left|\nabla \rho_{s}\right|^{2}\right] d V= \\
& \int_{\Omega}\left(\mathbf{b}_{s} \cdot \mathbf{v}_{s}+\mathbf{b}_{f} \cdot \mathbf{v}_{f}\right) d V+\int_{\partial \Omega}\left(\mathbf{t}_{s} \cdot \mathbf{v}_{s}+\mathbf{t}_{f} \cdot \mathbf{v}_{f}+\tau_{s} \cdot \frac{\partial \mathbf{v}_{s}}{\partial n}\right) d A
\end{aligned}
$$

where $\mathbf{F}_{s}$ is the deformation gradient for the solid, $\lambda_{s}>0$ is a material parameter with units of Newton (meter) ${ }^{6} / \mathrm{Kg}^{2}$, and $d_{\mathrm{v}} / d t$ signifies the material time derivative following the mean motion of the mixture. According to the Reynolds transport theorem the following constitutive equations must hold

$$
\begin{aligned}
\mathbf{T}_{s}= & \rho \frac{\partial \epsilon}{\partial \mathbf{F}_{s}} \mathbf{F}_{s}^{\top}-\lambda_{s}\left[\frac{f_{s s}}{2}\left(1+\xi_{f}\right) \mathbf{I}+\nabla \rho_{s} \otimes \nabla \rho_{s}\right] \\
p_{f}= & \rho \rho_{f} \frac{\partial \epsilon}{\partial \rho_{f}}-\frac{\lambda_{s}}{2} f_{s s} \xi_{f}, \\
\mathbf{\Pi}_{s}= & -\lambda_{s} \rho_{s} \mathbf{I} \otimes \nabla \rho_{s}, \\
\mathbf{m}_{s}= & -\rho\left[\xi_{f}\left(\nabla \mathbf{F}_{s}\right)^{\top} \frac{\partial \epsilon}{\partial \mathbf{F}_{s}}+\xi_{f} \mathbf{F}_{s}^{-\top} \frac{\partial \epsilon}{\partial X_{s}}-\xi_{s} \frac{\partial \epsilon}{\partial \rho_{f}} \nabla \rho_{f}\right. \\
& \left.+\frac{\lambda_{s}}{2 \rho} \nabla\left(\xi_{f} f_{s s}\right)\right]
\end{aligned}
$$

where $f_{s s}=\nabla \rho_{s} \cdot \nabla \rho_{s}$, and $\xi_{f}$ is the mass fraction of the fluid phase.

In this paper we limit our attention to external actions for which $\mathbf{b}_{s}=\mathbf{0}$ and $\mathbf{b}_{f}=\mathbf{0}$, i.e. only to external surface tractions. In order to find the partial tractions we assume the existence of a potential function such that the working, $\dot{W}^{\text {ext }}$, of external surface tractions is given by

$$
\dot{W}^{\mathrm{ext}}=\frac{d}{d t} \int_{\Omega} \psi^{\mathrm{ext}}\left(\mathbf{x}, \rho_{s}, \rho_{f}, \nabla \rho_{s}\right) d V
$$

where $\dot{W}^{\text {ext }}$ equals the surface integral on the right-hand side of eqn.(10). The external surface tractions for which eqn.(15) holds are conservative. Requiring 
that eqn.(15) hold for all choices of the velocity field, we obtain

$$
\begin{aligned}
& \frac{\partial \psi^{\mathrm{ext}}}{\partial \rho_{s}}-\operatorname{div}\left(\frac{\partial \psi^{\mathrm{ext}}}{\partial \nabla \rho_{s}}\right)=C_{s}, \quad \frac{\partial \psi^{\mathrm{ext}}}{\partial \rho_{f}}=C_{f}, \text { in } \Omega, \\
& \mathbf{t}_{s}=\left[-\frac{\partial \psi^{\mathrm{ext}}}{\partial \rho_{s}} \rho_{s}+\xi_{s} \psi^{\mathrm{ext}}-\frac{\partial \psi^{\mathrm{ext}}}{\partial \nabla \rho_{s}} \cdot \nabla \rho_{s}+\operatorname{div}\left(\rho_{s} \frac{\partial \psi^{\mathrm{ext}}}{\partial \nabla \rho_{s}}\right)\right. \\
& \left.+\rho_{s}\left(\frac{\partial \psi^{\text {ext }}}{\partial \nabla \rho_{s}} \cdot \mathbf{n}\right) \operatorname{tr}\left(\nabla^{s} \mathbf{n}\right)-\left(\frac{\partial \psi^{\text {ext }}}{\partial \nabla \rho_{s}} \cdot \mathbf{n}\right) \frac{\partial \rho_{s}}{\partial \mathbf{n}}\right] \mathbf{n} \\
& +\rho_{s} \nabla^{s}\left(\frac{\partial \psi^{\text {ext }}}{\partial \nabla \rho_{s}} \cdot \mathbf{n}\right) \text {, on } \partial \Omega \text {, } \\
& \mathbf{t}_{f}=\left(-\frac{\partial \psi^{\mathrm{ext}}}{\partial \rho_{f}} \rho_{f}+\xi_{f} \psi^{\mathrm{ext}}\right) \mathbf{n}, \text { on } \partial \Omega, \\
& \boldsymbol{\tau}_{s}=-\left(\rho_{s} \frac{\partial \psi^{\mathrm{ext}}}{\partial \nabla \rho_{s}} \cdot \mathbf{n}\right) \mathbf{n}, \text { on } \partial \Omega \text {. }
\end{aligned}
$$

Equations (16) with $C_{s}$ and $C_{f}$ as constants are necessary conditions for the existence of a $\psi^{\text {ext }}$ for which $\mathbf{b}_{s}=\mathbf{0}$ and $\mathbf{b}_{f}=\mathbf{0}$.

\section{Solution of a Boundary-Value Problem}

We analyze, within a linearized second gradient theory, the static infinitesimal deformations of an annular porous cylinder filled with an inviscid fluid and with the inner and the outer surfaces subjected to uniform external pressures $p_{1}^{\text {ext }}$ and $p_{2}^{\text {ext }}$ respectively. We assume that surface tractions on the inner and the outer surfaces of the cylinder, in the reference configuration, equal $-p_{0}$ and postulate that

$$
\begin{aligned}
\epsilon= & \frac{1}{\rho_{0}}\left[-p_{0} \mathbf{I} \cdot \mathbf{H}_{s}+\gamma_{f}^{0} \Delta \rho_{f}+\frac{1}{2} \mathcal{C}\left[\mathbf{H}_{s}\right] \cdot \mathbf{H}_{s}-\frac{1}{4} p_{0} \mathbf{H}_{s} \cdot\left(\mathbf{H}_{s}-\mathbf{H}_{s}^{\top}\right)\right. \\
& \left.+\frac{1}{2} \gamma_{f f}\left(\Delta \rho_{f}\right)^{2}+\Delta \rho_{f} K_{s f} \mathbf{I} \cdot \mathbf{H}_{s}\right],
\end{aligned}
$$

where $\mathbf{H}_{s}:=\nabla \mathbf{u}_{s}$ and $\Delta \rho_{\alpha}=\rho_{\alpha}-\rho_{\alpha}^{0}, \alpha=s, f ; \rho_{\alpha}^{0}$ is the mass density of the $\alpha$-th constituent in the reference configuration, $\mathcal{C}$ is the elasticity tensor, for the solid constituent, $\gamma_{f}^{0}, \gamma_{f f}$ and $K_{s f}$ are material parameters. The spherical tensor $K_{s f} \mathbf{I}$ accounts for the interaction between the solid and the fluid phases because of the deformations of the pores. The following form for the elasticity tensor is prescribed: $\mathcal{C}\left[\mathbf{E}_{s}\right]=\lambda \operatorname{tr}\left(\mathbf{E}_{s}\right) \mathbf{I}+2 \mu \mathbf{E}_{s}$, where $\lambda$ and $\mu$ are the Lamé.

We assume that deformations of the mixture are axi-symmetric, i.e., in cylindrical coordinates the two in-plane components of the displacement, $u_{r}$ and $u_{\theta}$, are functions of the radial coordinate $r$ only. In order to find boundary 
conditions on the solid and the fluid phases, we consider only those external tractions for which the following expression for $\psi^{\text {ext }}$ holds:

$$
\psi^{\mathrm{ext}}=C_{s} \rho_{s}+C_{f} \rho_{f}+\mathbf{C}_{\mathrm{int}}(r, \theta) \cdot \nabla \rho_{s} \Delta \rho_{s}+\hat{\psi}(r),
$$

$\hat{\psi}(r)=p_{0}+p_{1} r$ so that different tractions applied on the inner and the outer surface can be considered. Because of our interest in studying infinitesimal deformations, we retain terms bilinear in $\Delta \rho_{s}$ and $\nabla \rho_{s}$.

\section{Influence of coupling coefficient $K^{s f}$ on density profiles}

Through these assumptions the governing equations can be reduced to the following two uncoupled ordinary differential equations $\left({ }^{\prime}=\partial / \partial r\right)$ :

$$
\begin{gathered}
-\lambda_{s} \rho_{s}^{0^{2}}\left[U_{r}^{\prime \prime}+\frac{1}{r} U_{r}^{\prime}\right]+q\left(K_{s f}\right) \quad U_{r}=\Gamma_{s}, \quad U_{\theta}^{\prime}+\frac{2}{r} U_{\theta}=0 \\
q\left(K_{s f}\right)=2 \mu+\lambda-2 \xi_{f}^{0} p_{0}-\frac{\left(\xi_{s}^{0} \gamma_{f}^{0}+p_{0} / \rho_{0}-K_{s f}\right)^{2}}{2 \gamma_{f}^{0} / \rho_{0}+\gamma_{f f}}
\end{gathered}
$$

where $\Gamma_{s}$ is an integration constant to be determined by boundary conditions, $U_{r}=\left(u_{r}^{\prime}+1 / r u_{r}\right)=\operatorname{tr} \mathbf{H}_{s}$ and $U_{\theta}=\left(u_{\theta}^{\prime}-1 / r u_{\theta}\right)$ are dimensionless quantities; in particular $U_{r}$ is related to the increment of the solid apparent density by $\Delta \rho_{s}=-\rho_{s}^{0} U_{r}$. We specify boundary conditions coming from eqns.(7)-(9) and eqns.(17)-(19). Equation (22) $)_{1}$, for $\Gamma_{s}=0$, (homogeneous equation) belongs to the family of Classical or Modified Bessel equations, according to the sign of the coefficient $q$. Therefore two different solutions for the increment of the solid apparent density are obtained in dependence of $K_{s f}$ : if $q>0$ then the solution of eqn. $(22)_{1}$ is given by a linear combination of Modified Bessel functions $I_{0}(\xi)$ and $K_{0}(\xi)$, conversely if $q<0$ it is given by a linear combination of Classical Bessel functions $J_{0}(\xi)$ and $Y_{0}(\xi)$. In particular we notice that when $K_{s f} \in\left(K_{s f}^{(1)}, K_{s f}^{(2)}\right)$ the solution of the homogeneous equation is given by a linear combination of the Modified Bessel functions, as $\operatorname{sign}(q)=1$; conversely when $K_{s f} \in\left(-\infty, K_{s f}^{(1)}\right) \cup\left(K_{s f}^{(2)}, \infty\right)$ the solution of the homogeneous equation is a linear combination of the Classical Bessel functions, as $\operatorname{sign}(q)=-1 ; K_{s f}^{(1)}$ and $K_{s f}^{(2)}$ being the values of the coupling coefficient for which $q$ vanishes. The solution of eqn. $(22)_{1}$ is obtained by simply adding a suitable cone stant to solution of the homogeneous equation. In the following figures we draw plots of the $\Delta \rho_{s}$-profiles, for a damaged salt matrix filled with brine; values of constitutive and geometric parameters and surface tractions are listed in Table 1.

Note that values for the constitutive coefficients $\lambda_{s}$ and $C_{\text {int }}$ are introduced without any experimental validation of the model. However, this choice of 
Table 1. In the first column $E$ and $\nu$ are the Young modulus and the Poisson ratio of the solid matrix; in the second one $\hat{\rho}_{s}^{0}$ and $\hat{\rho}_{f}^{0}$ are the densities of the solid and the fluid constituent in the reference configuration, $\nu_{s}^{0}$ and $\nu_{f}^{0}$ their volume fractions (in the reference configuration the mixture is saturated).

\begin{tabular}{lcr}
\hline Constitutive parameters & Referential state parameters & Tensions \\
\hline$E=200 \mathrm{MPa}$ & $\hat{\rho}_{s}^{0}=1850 \mathrm{Kg} / \mathrm{m}^{3}$ & $p_{01}^{\mathrm{ext}}=20 \mathrm{MPa}$ \\
$\nu=0.33$ & $\hat{\rho}_{f}^{0}=1300 \mathrm{Kg} / \mathrm{m}^{3}$ & $p_{02}^{\mathrm{ext}}=20 \mathrm{MPa}$ \\
$\lambda_{s}=200 \mathrm{Nm} \mathrm{m}^{4} / \mathrm{Kg}^{2}$ & $\nu_{s}^{0}=0.97$ & $\tilde{p}_{0}=-2.21 \mathrm{MPa}$ \\
$\gamma_{f f}=1.6410^{6} \mathrm{Nm}^{4} / \mathrm{Kg}^{2}$ & $\nu_{f}^{0}=1-\nu_{s}^{0}=0.03$ & $p_{1}=10^{5} \mathrm{~N} / \mathrm{m}^{3}$ \\
$C_{s}=C_{f}$ & $\rho_{s}^{0}=\hat{\rho}_{s}^{0} \nu_{s}^{0}=1794.5 \mathrm{Kg} / \mathrm{m}^{3}$ & \\
$C_{\mathrm{int}}=1 \mathrm{Nm} \mathrm{m}^{3} / \mathrm{Kg}^{2}$ & $\rho_{f}^{0}=\hat{\rho}_{f}^{0} \nu_{f}^{0}=39 \mathrm{Kg} / \mathrm{m}^{3}$ & \\
& $R_{1}=2 \mathrm{~m}, \quad R_{2}=20 \mathrm{~m}$ & \\
& &
\end{tabular}

values is based on the expectation that these values can describe the poreopening effect close to the boundary of the mixture.

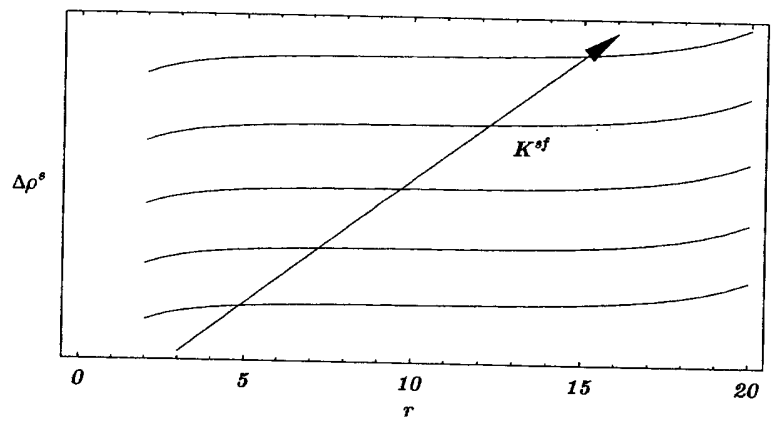

Figure 1. Qualitative $\Delta \rho_{s}$-profiles for $K_{s f} \in\left(K_{s f}^{(1)}, K_{s f}^{(2)}\right)$.
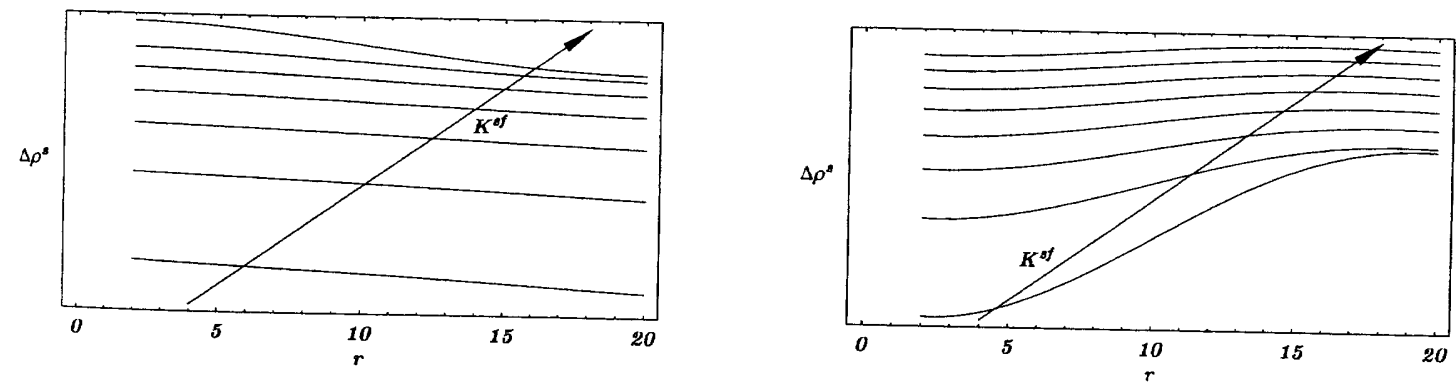

Figure 2. Qualitative $\Delta \rho_{s}$-profiles for $K_{s f} \in\left(-\infty, K_{s f}^{(1)}\right) \cup\left(K_{s f}^{(2)}, \infty\right)$. Figure $2 a$ corresponds to $1.75<K<1.81$, and Figure $2 b$ to $1.83<K<1.9$.

In Figure 1 the typical behavior of fields exhibiting boundary layers is shown, in Figure 2 the solution apparently shows wide oscillations due to the 
change of type occurring in the Bessel equation; this is usually an indication of instability.

On the basis of the previous remarks on admissible values of the coupling coefficient $K_{s f}$ we wish to establish which conditions assure the uniqueness of the solutions of the elastic problem. In doing this our aim is therefore the characterization of those coupling parameters which guarantee the so-called structural stability of the PDEs (defined on the space of the considered state parameters $u_{s}$ and $\Delta \rho_{f}$ ) which describe the governing equations of the mixture. According to the criterion stated by [1], we discuss the possibility that for two different representative elements of this family, corresponding to sufficiently close values of $K_{s f}$, an homeomorphism on the space of state parameters exists transforming one solution into the other (topological equivalence). In order for the aforementioned PDEs to fulfill this requirement it is assumed that the trajectories of a representative element of the vector field family prescribed by eqns.(22) describes available transformations of a given reference configuration. Indeed we adopt a physically meaningful energetic criterion: the reference configuration is stable if the total energy

$$
\mathcal{E}_{\text {tot }}\left(\rho_{f}, \mathbf{F}_{s}\right)=\int_{\Omega}\left[\rho \epsilon\left(\rho_{f}, \mathbf{F}_{s}\right)+\frac{\lambda_{s}}{2}\left|\nabla \rho_{s}\right|^{2}-\psi^{\mathrm{ext}}\left(\mathbf{x}, \rho_{s}, \rho_{f}, \nabla \rho_{s}\right)\right] d V
$$

attains its minimum in the reference configuration. In particular we prove structural stability when the coupling coefficient lies in a suitable subset of the open interval $\left(K_{s f}^{(1)}, K_{s f}^{(2)}\right)$. The second functional derivative of eqn.(24), evaluated in the reference configuration, is in this case positive definite.

In order to prove this statement we assume the second functional derivative of $\mathcal{E}_{\text {tot }}$ evaluated in the reference configuration to equal the integral over $\Omega$ of a suitable quadratic form multiplied by a scalar quantity $\alpha$ and determine under which conditions the corresponding spectral problem admits positive eigenvalues. In particular the following quadratic form is assumed for the second functional derivative of $\mathcal{E}_{\text {tot }}$

$$
\left.\frac{d^{2} \mathcal{E}_{t o t}}{d t^{2}}\right|_{\left(\rho_{f}^{0}, \rho_{s}^{0}\right)}=\int_{\Omega} \alpha\left[\left(\operatorname{div} \mathbf{v}_{s}\right)^{2}+\operatorname{skw}\left(\nabla \mathbf{v}_{s}\right) \cdot \operatorname{skw}\left(\nabla \mathbf{v}_{s}\right)+\left(\operatorname{div} \mathbf{v}_{f}\right)^{2}\right] d V
$$

Numerical simulations show that structural stability is guaranteed in this case for values of the coupling coefficient which belong to a suitable open subset $\left(K_{s f}^{1 \mathrm{~s}}, K_{s f}^{2 \mathrm{~s}}\right)$ of $\left(K_{s f}^{(1)}, K_{s f}^{(2)}\right)$. This means that the meaningful solutions of eqns.(22) are those for coupling coefficients belonging to this open interval only. 


\section{Concluding Remarks}

In this paper a static linear elastic deformation problem for a fluid saturated solid is formulated in which the behavior of the solid matrix is described by a second gradient model. The non-deformed configuration, chosen as a reference configuration, for the considered mixture can not be stress-free: indeed the saturating fluid must exhibit internal stresses acting both on the solid constituent and on its sub-bodies.

When limiting our attention to purely spherical pre-stress we find analytical forms for the solutions of Bessel or Modified Bessel equations in dependence on the coupling coefficient $K_{s f}$. The obtained density profiles may show an oscillating behavior; we prove the conjecture that oscillating profiles are unstable as well as the non-oscillating ones which correspond to sufficiently high absolute values of $K_{s f}$.

\section{References}

[1] Arnold, V.I. (1983) Geometrical methods in the theory of ordinary differential equations; translated by Joseph Szucs; English translation edited by Mark Levi. Springer, New York

[2] Biot, M.A. (1941) General theory of three-dimensional consolidation, J. Applied Physics 12, 155-164

[3] Cosenza, P., Ghoreychi, M., Bazargan-Sabet, B. and de Marsily, G. (1999) In situ rock salt permeability measurement for long term safety assessment of storage, Int. J. Rock Mech. Min. 36, 509-526

[4] dell'Isola, F. and Seppecher, P. (1997) Edge contact forces and quasi balanced power, Meccanica 32, 33-52

[5] Elhers, W. (1991) Toward finite theories of liquid-saturated elasto-plastic porous media, Int. J. of Plasticity 7, 433-475

[6] Fillunger, P. (1936) Erdbaumechanik?, Selbstverlag des Verfassers, Wien

[7] Germain, P. (1973) La méthode des puissances virtuelles en mécanique des milieux continus, Journal de Mécanique 12(2), 235-274

[8] Germain, P. (1973) The method of virtual power in continuum mechanics. Part 2: microstructure, SIAM J. Appl..Mech. 25(3), 556-575

[9] Rajagopal, K.R. and Tao, L. (1995) Mechanics of Mixtures, World Scientific

[10] Truesdell, C. (1957) Sulle basi della termomeccanica, Lincei - Rend. Sc. fis. mat. e nat. Vol. XXII 